Longitudinal Stochastic Cooling Power Requirements:

$\underline{\text { A Coasting-Beam Approach }}$

J. Wei, M. Blaskiewicz, M. Brennan

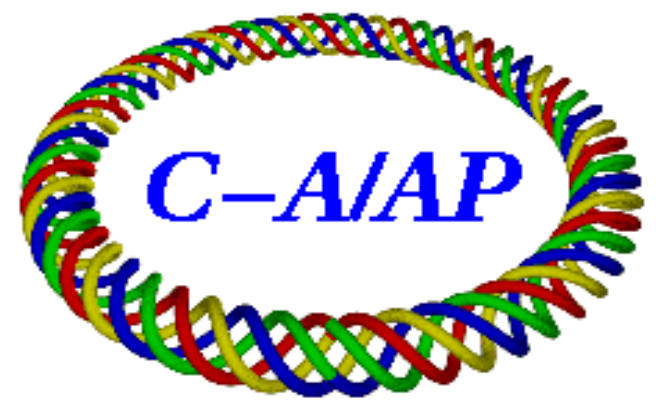

Collider-Accelerator Department Brookhaven National Laboratory

Upton, NY 11973 


\title{
Longitudinal Stochastic Cooling Power Requirements: A Coasting-Beam Approach
}

\author{
J. Wei, M. Blaskiewicz, M. Brennan \\ Brookhaven Nalional Laboralory, Uplon, New York 11973, U.S.A.
}

\begin{abstract}
$\Lambda$ practical obstacle for stochastic cooling in RHIC is the large amount of power needed for the cooling system. This paper discusses the cooling power needed for the longitudinal conling process. Based on the coasting-beam Fokkcr-Planck equation, we analytically derived the optimum cooling ratc and cooling power for a beam of uniform distribution and a cooling system of linear gain function. The results indicate that the usual back-of-envelope formula ovcr-cstimated the cooling powcr by a factor of the mixing factor $M$. On the other hand, the scaling laws derived from the coasting-beam Fokker-Planck approach agree with those derived from the bunched-beam Fokker-Planck approach if the peak beam intensity is used as the effective coasting-beam inlensily. A longiludinal stochastic cooling system of $4-8$ GHz bandwidth in RHIC can effectively counteract intrabeam scattering, preventing the beam from escaping the RF bucket becoming debunched around the ring.
\end{abstract}




\section{INTRODUCTION}

Stochastic cooling [1,2] has long been recognized as a viable approach to counteract the emittance growth and beam loss caused by intrabeam scattering in RHIC [3,4]. Theoretically, with a transverse cooling system of frequency bandwidth from 4 to $8 \mathrm{GHz}$, the (nornalized 95\%) entitlance of a gold bean of $10^{9}$ particles per bunch can be preserved at $30 \pi \mu \mathrm{m}$. With a longitudinal cooling system of the same frequency bandwidth, the beam escaping from the RF bucket debunched around the ring can be climinated [5].

A possible technical obstacle is the existence of very strong coherent components at $\mathrm{GH} z$ frequency range that would saturate the electronics of the cooling system and swamp the true stochastic information. Duc to this problem, attempts at implementing bunched-bcam stochastic cooling at the 'levatron and the SPS were unsuccesslul. On the other hand, cooling of the heavy ion beam in RHIC has the advantage that the signal-to-noise ratio is high due to the high charge state, and that longitudinally the bcam occupics a large fraction of the Rr bucket. Recent measurements of Schottky signals of the gold bean indicate that stochastic cooling in RHIC would not be impeded by anomalous coherent components in the Schottky signals $[6,7]$.

Practically, the obstacle for stochastic cooling in RHIC is the large amount of power needed for the cooling system [3]. Early study using the Fokker-Planck approach indicated that the power needed is proportional to the encrgy spread of the beam to the forth power [4]. With a total kicker coupling-resistance of $6.4 \mathrm{k} \Omega$, the power needed for longitudinal cooling at beam storage is several kilo Watts at a frequency range from 4 to $8 \mathrm{GHz}$. However, a comparison between the Fokker-Planck calculation $\mid 4\rfloor$ and the estimate given in Ref. [3] indicates a difference in the scaling behavior of the cooling power when the mixing factor [?] is larger than 1unity. The purpose of this note is to present the analytical derivation of the cooling power scaling law and to discuss applications in RHIC. 


\section{FOKKER-PLANCK APPROACH}

\section{A. Formalism}

Define the quantity $W$ as

$$
W \equiv \frac{\Delta E}{\omega_{s}}
$$

where $\omega_{s}$ is the revolution frequency and $\Delta E$ is the deviation in energy. Assume that the evolution of the beam distribution is slow during a synchrotron oscillation period. The evolution of the longitudinal density lunction $\psi(W)$ can be described by the Fokker-Planck equation $[8,9,2]$

$$
\frac{\partial \Psi}{\partial t}=-\frac{\partial}{\partial W}(F \Psi)+\frac{1}{2} \frac{\partial}{\partial W}\left[\left(D_{s h}+D_{t}\right) \frac{\partial \Psi}{\partial W}\right]
$$

The boundary condition of Fq. 1 is that $\Psi$ vanishes at the energy aperture $W_{\max }$ and the flux vanishes at $W=0$,

$$
\begin{cases}-F \Psi+\frac{1}{2}\left(D_{s h}+D_{t}\right) \frac{\partial \Psi}{\partial W}=0 & W=0 \\ \Psi=0 & W=W_{\max }\end{cases}
$$

The drifting coefficient $F$ corresponds to the coherent component of the cooling force, and the diffusion coefficients $D_{s h}$ and $D_{t}$ correspond to Schottky noise and system thermal noise,

$$
\begin{gathered}
F(W)-\lim _{\Delta t>0} \frac{1}{\Delta t}\left\langle\int_{0}^{\Delta t} d t U_{W}(t)\right\rangle \\
D_{s h}(W)-\lim _{\Delta t>0} \frac{1}{\Delta t}\left\langle\int_{0}^{\Delta t} d t \int_{0}^{\Delta t} d t^{\prime} U_{W}(t) U_{W}\left(t^{\prime}\right)\right\rangle
\end{gathered}
$$

Thermal noise is usually small for cooling of heavy ion beams and thus neglected in this dis cussion. The energy kick $U_{W}$ applied on a test particle $i$ by the cooling system contains both the deterministic (coherent) part resulting from its own signal and a fluctuating (incoherent) part resulting from the signal generated by all other particles.

$$
U_{W, i}-\frac{Z e}{\omega_{s}} \sum_{n=-\infty}^{\infty} V_{k}(t) \delta\left(t-\frac{2 \pi n}{\omega_{i}}-\frac{\phi_{i}}{\omega_{i}}-\frac{\theta_{k}}{\omega_{i}}\right)
$$


where $\phi_{i}$ is the phase of the test particle, $\theta_{k}$ is the azimuthal location of the kicker in the ring, the subscript $k$ indicates the kicker, and

$$
V_{k}(t)=\frac{1}{2 \pi} \int_{-\infty}^{\infty} G(\omega) I_{p^{m}} \exp (i \omega t) d \omega
$$

where $G(\omega)$ is the gain function, and the frequency component of the beam current is given by

$$
\begin{gathered}
I_{p u}(\omega)=\int_{-\infty}^{\infty} I_{p u} \operatorname{cxp}(i \omega t) d t \\
I_{p u}(t)=Z_{c} \sum_{m=\infty}^{\infty} \sum_{j=1}^{N} \delta\left(\begin{array}{cccc}
2 \pi m & \phi_{j} & \theta_{p u} \\
\omega_{j} & \omega_{j} & \omega_{j}
\end{array}\right),
\end{gathered}
$$

where the subscript $p u$ indicates the signal detector pick-up. Use the relation on the gain function imposed by the causality condition

$$
G(-\omega)=G^{*}(\omega)
$$

and mathematical relations

$$
\begin{gathered}
\sum_{m--\infty}^{(\infty)} \exp -i \frac{2 \pi m \omega}{\omega_{i}}=\omega_{i} \sum_{m--\infty}^{(\infty)} \delta\left(\omega-m \omega_{i}\right) \\
\lim _{\Delta t \rightarrow \Delta l} \frac{1}{\Delta t} \int_{0}^{\Delta t} d t \operatorname{cxp}(a t)= \begin{cases}1 & a=0 \\
0 & a \neq 0\end{cases}
\end{gathered}
$$

The drifting coefficient becomes

$$
F=\frac{z^{2} e^{2} \omega_{i}}{4 \pi^{2}} \sum_{m=-\infty}^{\infty} G\left(m \omega_{i}\right) \exp \left\lfloor-i m\left(\theta_{p u}-\theta_{k}\right)\right\rfloor
$$

where the factor $\exp \left[-i m\left(\theta_{p u}-\theta_{k}\right)\right]$ is the effect from the delay between the pick-up and the kicker, and the summation is over the effective frequency range of the cooling system. 'I'he diffusion coefficient becomes

$$
D_{s h}=\left.z^{4} e^{4} \omega_{i}^{2} \sum^{3} \sum_{j=1}^{N} \sum_{m=\infty}^{\infty} \sum_{n=\infty}^{\infty}\left|G\left(m \omega_{j}\right)\right|^{2} \rho\left(\omega_{j}\right)\right|_{\omega_{j}=\frac{n}{m} n_{i}}
$$


where the summation over $j$ indicates the contribution from all the particles, and the double summation over $m$ and $n$ considers the case of frequency overlapping.

The average power required for cooling is

$$
\bar{P}=\lim _{\Delta t \rightarrow 0} \frac{1}{\Delta t} \int_{0}^{\Delta t} d t \frac{\left\langle V_{k}^{2}(l)\right\rangle}{n_{k} R_{k}}=\frac{\left\langle V_{\text {cool }}\right\rangle^{2}}{n_{k} R_{k}}
$$

where $\left\langle V_{\text {cool }}\right\rangle$ is defined as the mean cooling voltage, $n_{k}$ is the number of kicker pairs, and $R_{k}$ is the coupling resistance of each kicker pair.

\section{SCAIING IAWS FOR COOLING RATE AND POWER}

'T'he cooling rate of the beam energy spread

$$
\langle W\rangle=2 \int_{0}^{W_{\max }} W \Psi(W) d W
$$

can be obtained from the Foller-Planck equation as

$$
\begin{gathered}
\partial\langle\bar{W}\rangle \\
\partial l
\end{gathered}=2 \int_{0}^{W_{\text {max }}} d W\left(F+\begin{array}{l}
1 \partial \bar{D} \\
2 \partial W
\end{array}\right) \Psi(W)
$$

To obtain a scaling of the cooling rate, we assume a linear gain function "notched" at multiples of the revolution frequency

$$
G(m \omega)=g m W, \quad \Delta \omega=\omega-m \omega_{s}=-\frac{\eta \omega_{s}^{2}}{E_{s} \gamma^{2}} W
$$

where $m \omega_{s}$ is the nearest multiple of the revolution frequency to $\omega, \eta=\frac{1}{\gamma_{t}^{2}}-\frac{1}{\gamma^{2}}$ is the slip factor, $E_{s}$ is the beam energy, $\gamma$ is the Lorentz factor, and $\gamma_{t}$ is the $\gamma$ value of the transition energy. Denote the eflective frequency range of cooling from $n_{1} \omega_{s}$ to $n_{2} \omega_{s}$, the drifting and diffusion coefficients becomes

$$
\begin{gathered}
F \approx \frac{Z^{2} e^{2} \omega_{s}}{2 \pi^{2}} \bar{n} \Delta n \exp \left[i \bar{n}\left(\theta_{k}-\theta_{p u}\right)\right] g W \\
D \approx \begin{array}{c}
Z^{4} e^{4} \omega_{s}^{2} \\
4 \pi^{3}
\end{array} \bar{n} \Delta n g^{2} W^{2} \rho\left(\omega_{i}\right)
\end{gathered}
$$


where $\Delta n=n_{2} \quad n_{1}, \bar{n}=\begin{gathered}n_{1}+n_{2} \\ 2\end{gathered}$, and the Schottky bands are assumed to be nonoverlapping. For a uniform density distribution

$$
\rho(W)=\left\{\begin{array}{cc}
N & |W|<W_{0} \\
2 \Delta \omega_{* s} & \\
0 & \text { otherwise }
\end{array}\right.
$$

where

$$
\Delta \omega_{s}=-\eta \omega_{s}(\Delta \hat{p} / p)_{0}=-\frac{\eta \omega_{s}^{2}}{E_{s} \gamma^{2}} W_{0}
$$

the maximum cooling rate that corresponds to the minimum cooling time $\tau_{\min }$ is obtained ds

$$
\tau_{m i n}^{1}=\left.\frac{1}{\langle W\rangle} \frac{\partial\langle W\rangle}{\partial t}\right|_{\max } \approx-\frac{\Delta n \omega_{s}}{2 \pi N M}
$$

where the "bad mixing" factor is neglected, and the mixing factor $M$ is given by

$$
M=\frac{1}{\bar{n}|\eta|(\Delta \hat{p} / p)_{0}}=\frac{1}{\sqrt{3} \bar{n}|\eta| \sigma_{p}}, \quad \text { for } \quad M>1
$$

where $\sigma_{p}$ is the rms spread in momentum $(\Delta p / p)$. The optimum gain function to achieve the maximum cooling rate is given by

$$
g_{o p t} \approx-\frac{2 \pi|\eta|(\Delta \hat{p} / p)_{0}}{Z^{2} e^{2} N}
$$

The average power needed for cooling is

$$
\bar{P} \approx \frac{2}{f_{s} n_{k} \bar{R}_{k}} \frac{1}{\tau_{\min } \bar{M}}\left(\frac{\beta^{2} E_{s} \sigma_{p}}{Z e}\right)^{2}
$$

where $f_{s}=\omega_{s} / 2 \pi$ is the revolution frequency.

\section{NUMERICAL EXAMPLE: LONGITUDINAL COOLING IN TRHIC}

Coasting-beam estimate of the cooling rate and cooling power is the following. Consider longitudinal stochastic cooling of a gold beam $(Z=79, A=197)$ at. RHIC storage $(\gamma=108)$ with $h=2520 \mathrm{RF}$ system of $6 \mathrm{MV}$ voltage, and a bunch of intensity $N_{0}=10^{9}$. The 
revolution frequency is $\omega_{s} / 2 \pi=78 \mathrm{kIIz}$. The slip factor is $\eta=1.9 \times 10^{-3}$. The central harmonic corrsponding to a $4 \quad 8 \mathrm{GHz}$ systom is $\bar{n}=7.7 \times 10^{4}$. During a 10 -hour store under intrabeam scattering and longitudinal stochastic cooling [10], the bunch length $\sigma_{l}$ varies from about $0.11 \mathrm{~m}$ to $0.19 \mathrm{~m}\left(\sigma_{\phi}\right.$ varies from about $27^{\circ}$ to about $\left.45^{\circ}\right)$, and the bunching factor $B$ varies from 0.19 to 0.31 . With cooling, the reduction in beam intensity is small during the store, and the effective number of particle in the ring $N=h N_{0} / B$ varies from about $1.33 \times 10^{13}$ to about $0.81 \times 10^{13}$. The rms momentum spread $\sigma_{p}$ varies from about $0.42 \times 10^{3}$ to about $0.71 \times 10^{3}$, and the mixing factor $M$ varies from 9.4 to 5.6 (Eq. 21). According to Eq. 20, the optimum cooling time varies from 8.7 to 3.2 hours. The mean cooling voltage $\left\langle V_{\text {cool }}\right\rangle$ varies from about $1.0 \mathrm{kV}$ to about $3.6 \mathrm{kV}$ (Eq. 14). Assume $n_{k}=128$ pairs of kickers cach at $R_{k}=50 \Omega$ coupling resistance. The average cooling power varies from $0.15 \mathrm{~kW}$ lo $2.0 \mathrm{~kW}$ (Eq. 23). The peak power is proportional to the 1th power of the momentum spread $\sigma_{p}$.

\section{DISCUSSIONS AND SUMMARY}

Based on the coasting-beam Fokker-Planck equation, we analytically derived the optimum cooling rate and cooling power for a beam of uniform distribution and a cooling system of a linear gain function. The results indicate that the usual back-of-envelope formula $[3\rfloor$ over-estimated the cooling power by a factor of the mixing factor $M$. On the other hand, the scaling laws derived from the coasting-beam Fokker-Planck approach agree with those derived from the bunched beam Fokker Planck approach $[4\rfloor$ if the peak beam intensity is used as the effective coasting-beam intensity. Although we have ignored signal suppression for the entire discussion, the conclusion holds.

A longitudinal stochastic cooling system of $4-8 \mathrm{GHz}$ bandwidth in RHIC can effectively counteract intrabeam scattering, preventing the gold beam from escaping the RF bucket becoming debunched around the ring. Combining with a transverse stochastic conling system of the same frequency bandwidth to contain the transverse emittance, we expect a significant 
increase in the average luminosity.

\section{ACKNUWLEDGMEN'TS}

We would like to thank J. Marriner for many uselul discussions. 


\section{REFERENCES}

[1] S. van der Meer, Stochastic damping of betatron oscillations in the ISR, CERN/ISR$\mathrm{PO} / 7231$ (1972)

[2] D. Möhl, G. Petrucci, T. Thorndahl, and S. van der Meer, Physics and technique of stochastic cooling, Physics Reports, 58, No. 2 (1980) 73.

[3] S. Van der Mcer, Stochastic cooling in RHIC, BNL technical note RHIC-AP-9 (1984)

[4] J. Wei, A.G. Ruggiero, Longitudinal stochastic cooling in RHIC, BNL technical note AD/RHIC-71 (1990)

[5] .J. Wei, Stochastic cooling and intrabeam scattering in RHIC, Workshop on beam cooling and related topics, CERN 94-03 (1991), edited by J. Bosser, p. 132

[6] J.M. Brennan, M. Blaskicwicz, P. Camcron, J. Wei, Observations of Schottky signals in RHIC and lheir polential Jor slochaslic cooling, European Parlicle Acceleralor Conlerence, Paris (2002), p. 308

[7] M. Blaskiewicz, J.M. Brennan, P. Cameron, J. Wei, Stochastic cooling studies in RIIIC, Particle Accelerator Conference, Portland (to be published, 2003).

[8] F. Sacherer, Stochastic cooling theory, CERN-ISR-TII/78-11 (1978).

[9] S. Chatiopadhyay, On slochuslic cooling of bunched beams from fluclualion and kinelic theory, LBL-14826 (1982).

[10] J. Wei, results based on calculation using computer program BBFP with bunched-beam Fokker-Planck equation solver, 2003. 